\title{
Coalition-Oriented Sensing in Wireless Sensor Networks
}

\author{
M. del Carmen Delgado-Roman and Carles Sierra
}

Artificial Intelligence Research Institute (IIIA), Spanish Scientific Research Council (CSIC), Universitat Autònoma de Barcelona, Bellatera E08193, Barcelona, Spain

\{delgado, sierra\}@iiia.csic.es

\begin{abstract}
Wireless Sensor Networks are generally composed of a large number of nodes that monitor their surrounding area. The monitoring capacity of sensors gets altered by the changing conditions of the environment and the sensors' internal state. Sensor coalitions, in which only the leader transmits information to a sink node, are a means to save resources when the conditions of the environment are similar around the sensors in the coalition. In this paper we analyse and formalise such sensor coalitions and propose an algorithm for coalition formation that allows the sensors to self-organise with the purpose of performing a good monitoring of the environment while maximising the life span of the sensor network as a whole. The algorithm uses the quality of the information fused at the coalition leader and the remaining energy of the sensors as the basic parameters to alter coalition membership and leadership.
\end{abstract}

Keywords: Wireless Sensor Networks, Sensor Coalitions, Resourse Saving Strategies.

\section{Introduction}

Wireless Sensor Networks (WSN) are becoming widespread thanks to the advances in electronics and wireless communication [1]. Nowadays, there is a plethora of small low-cost and low-energy consuming sensors able to communicate via wireless technology. These devices have made many monitoring tasks simpler and cheaper. Typical applications include environment monitoring, security control, military surveillance or traffic control.

The main challenge that WSNs put to scientists is the management of the hard constraints imposed on the sensors, like low communication bandwidth, little processing capacity and limited availability of energy. These restrictions make it necessary to use a large number of sensors such that there is always a minimum number of operational sensors to do the task. Multiagent system technologies can alleviate such constraints by introducing coordination between sensors to improve the performance of the network. The problem tackled in this paper is the development of energy-saving data treatment strategies based on the local activity of nodes in a WSN. In a generic scenario, the task of a sensor is to sense the environment and send the collected data to a server node, the

D. Kinny et al. (Eds.): PRIMA 2011, LNAI 7047, pp. 448 459, 2011.

(C) Springer-Verlag Berlin Heidelberg 2011 
sink. Although WSNs are typically deployed in dynamic environments, periods of stable conditions may not be infrequent. These periods do not require high sensing rates as this would entail transmitting the same data every time. The same argument holds in space: sensors situated close to one another may collect similar data. This phenomenon can be used to check the spatial coherence of the collected data and to save nodes' energy by avoiding the transmission of repeated samples. The inspirational scenario considered for this algorithm proposal is that of a WSN deployed over a waterway to monitor its state.

The distributed algorithm proposed in this paper implements a strategy for (not necessarily optimal) coalition formation in WSNs that trades-off information-gain and individual nodes' energy. The algorithm is fully distributed and embedded in the sensors functioning regime and we assume that coalitions don't serve the individual goals of agents but a shared common goal. The network's life span is increased at the cost of sending less data to the sink. The balance between quality of information collected and available energy is what determines the network division into coherent coalitions of agents.

In this paper we make two contributions. First, we formalise the problem of environmental monitoring in WSN through the network division into groups. Secondly, we propose an algorithm for coalition formation that allows for a tunable trade-off between information-gain at the sink and life-span of the whole network. To our knowledge no previous works followed this approach.

The rest of the paper is organised as follows. In Section 2, we briefly revise previous contributions to the important field of coalition formation in Multiagent Systems. Next, a formal description of the problem studied is presented in Section 3. Section 4 presents a detailed description of the algorithms designed and finally, we draw some conclusions and discuss future work in Section 5.

\section{Related Work}

Multiagent Systems (MAS) are composed of distributed autonomous entities that have to coordinate themselves to solve a common task. A simple coordination mechanism for agents is to organise themselves in coalitions and cooperate to share resources or reach goals that cannot be achieved individually. From a MAS perspective, coalitions represent a fundamental form of organisation.

According to the classical formulation, a coalition is defined as a set of selfinterested agents that cooperate to achieve a common goal. Agents try to maximise their individual and groupal payoff while guaranteeing coalition stability. According to 14, Coalition Formation (CF) in MAS can be studied from three different perspectives:

- Task allocation. Many MAS applications require agents to join forces for a period of time to solve a task. Contract Net Protocol [13] represents one of the first proposals in this line.

- Social networks. This research line uses coalitions to study the emergence and behaviour of organisations in environments without clearly defined interaction mechanisms [4. 
- Game theory. This approach to CF does not focus on the design of agents' strategies to reach beneficial coalitions [14] but on the coalitions stability.

$\mathrm{CF}$ in its traditional view, as a static and centralized problem, is NP complete [10. However MAS and WSN do not satisfy this view as their application environments are typically dynamic and distributed. Hence, a number of alternative mechanisms for coalition formation in this kind of environments have been proposed in recent years.

A CF protocol for task accomplishment in situations of incomplete information was proposed in [8]. In the studied scenario, agents collaborate and reach decisions for task completion with incomplete information about the environment and the other agents. The algorithm is based on a negotiation process developed for a so called Request For Proposals domain. In the considered scenarios, business agents tackle complex decomposable tasks that require the formation of groups of provider agents to solve them.

Task oriented $\mathrm{CF}$ in dynamic environments faces the problem of high power and bandwidth consumption due to continuous configuration and reconfiguration processes to adapt to evolving system conditions and demands. To avoid that excessive consumption, [2] proposes a task oriented CF in which the coalition duration is calculated following some fuzzy rules applied to the historical behaviour of the agents and the characteristics of the tasks arriving to the system.

The previously presented approaches do not pay attention to individual agents cooperative or self-interested will. This dimension was added to the problem through the concept of clan [7. A clan designates a set of agents that have similar aims and who also trust each other. In this case, group formation is not only determined by task accomplishment, but by the agents' motivation and trust relationships, originating mid-term duration coalitions.

The application of CF techniques to sensor networks has also been investigated by numerous researchers. For instance, a vehicle-tracking sensor network is modelled using disjoint coalitions of homogeneous agents in [12]. Coalitions are formed via a negotiation process based on local and social marginal utility calculations that take place in an incomplete information scenario. To maximise the system's performance, the proposed algorithm enables the self-organisation of the system by allowing the agents to discover their organizational relationships during the negotiation process. As a result, CF can also be observed as an organization method in MAS, as it naturally fits within the structure of a system without a central authority.

Assuming that sensor networks should be inherently adaptive, 9] proposed the Dynamic Regions Theory, whose objective is to optimize the overall operation of the network through its own partition into several regions that execute different algorithms. The network partition is derived from the individual nodes' role election according to their current circumstances and the system global policy. The goodness of this approach is shown for a specific gas plume detection scenario. A new dimension was added to the problem of CF in [5]: the study of the influence of the network topology structure in a MAS perfomance for task solving. The system divides itself into disjoint groups to accomplish the 
demanded tasks. During the execution, agents can rewire their connections to form better coalitions according to a degree of connectivity or a performancebased policy. A new network adaptation policy for the same situation was introduced in [3]. In this case, agents rewire the network according to their similarity to their neighbours. They also choose to which group they want to adhere and when to leave it based on some task and group success indicators. In the same line, 6] enriched the previous situation by considering a more realistic coalition model. The two rewiring policies developed in the system were based on performance and a different similarity definition. As in previous approaches, the implementation of these policies makes the system outperform an initial situation without rewiring capacity. However, none of these three approaches takes into account the energy consumption and cost derived from the rewiring pocilies.

Guided by the same objective of extending the lifetime of a glacial sensor network, 11] proposes an algorithm for adaptive sampling. Nevertheless, in contrast with our approach, nodes there follow an individual policy to reach the desired objective, while we focus on groupal strategies to save energy in sampling tasks.

In this paper, we propose a CF strategy for homogeneous nodes in a sensor network scenario. The sensor nodes' task is to monitor the behaviour of the environment in which they are deployed. In contrast with previous work, the CF strategy aims at saving energy to extend the network lifetime. This is achieved by allowing nodes in a coalition to delegate their sensing tasks to other neighbouring nodes, while restricting the maximum information loss so that the initial purpose of the system — faithfully monitoring the environment - is not missed.

\section{Problem Formalisation}

A WSN is composed of an initial set of cooperative and homogeneous nodes, from now on agents. Each of these agents has the same sensing capability, so they all can sample the variable $x$ being observed at any time $t$. The basic behaviour of an agent consists of sensing the environment and sending this information to a server or sink. We will note by $A=\left\{a_{1}, \ldots, a_{i}, \ldots, a_{N}\right\}$ the set of sensing agents and by $a_{s}$ the sink agent, $a_{s} \notin A$.

To save system resources, agents will organise themselves into disjoint groups. Nodes in a group accomplish their tasks together as an entity, avoiding redundant sensing by the members of the group and unnecessary routing among them. In this way, we will save energy from the batteries of the sensors. To find an appropriate division of the agents at time $t$, we take into account the similarity of the individual measurements and the topology of the neighbourhood structure. The unit distance assumed for this scenario is one radio hop. Let $d: A \times A \rightarrow$ $\mathbb{N}$, be the distance between two nodes, measured as the minimum number of radio hops between them. The physical properties of wireless communication guarantees that $d$ is a metric distance. In particular, $d$ is commutative, $d\left(a_{i}, a_{j}\right)=$ $d\left(a_{j}, a_{i}\right)$, and $d\left(a_{i}, a_{i}\right)=0$.

Now we can define the notion of neighbourhood. Based on $d$, and given a set of agents $A$, we call $N e: A \rightarrow 2^{A}$ a neighbourhood function if and only if $a_{j} \in N e\left(a_{i}\right) \Leftrightarrow d\left(a_{j}, a_{i}\right)=1$. 
The coalition structure is then defined for a maximum distance $\alpha$ among its members. The $\alpha$ parameter influences the maximum achievable size of the groups in a coalition, and hence the minimum granularity of the network. Given a set of agents $A$, an $\alpha$-distance coalition structure, $c_{\alpha}=\left\{g_{k}\right\}_{k: 1 . . K}$, is a partition of $A$ in $K$ groups, such that $\forall a_{i}, a_{j} \in g_{k}, d\left(a_{i}, a_{j}\right) \leq \alpha$. We note by $C_{\alpha}$ the set of all possible $\alpha$-distance coalition structures and the current coalition structure at time $t$, as $c^{t}$.

The criterion that guides the formation of the different coalition structures is to find (in a distributed manner) the best partition so that the quality of the information sent to the sink is somehow maximised and the energy consumption of the system somehow minimised. For such scenario, we propose the Coalition Oriented Sensing Algorithm (COSA), a tuneable algorithm able to fulfill these requirements. The adaptability of COSA is achieved through the definition of a set of parameters $p$ (to be explained later) whose values are going to drive the agents' behaviour. For a certain $p$ parameters configuration, agents take different kinds of sampling and transmission actions, represented as $m^{j} \in M_{p}$, where $M_{p}$ is the set of existing actions available for that $p$ configuration. The objective of minimising the system's energy consumption is formally expressed in the first part of (1). According to this, we try to find an optimal set of parameters $p *$, where $m_{i}^{j}$ is the action $j$ taken by agent $i$ and $E_{j}$ represents the energy consumption associated to that action.

$$
p *=\underset{p \in P}{\arg \min } \Delta E=\underset{p \in P}{\arg \min } \sum_{m^{j} \in M_{p}} \sum_{a_{i} \in A} \# m_{i}^{j} E_{j} ; \quad \text { s.t. } \quad Q o I(t) \geq \epsilon \quad \forall t .
$$

The identification of $p *$ is subject to guaranteeing that the Quality of Information received at the sink (QoI) is over a certain threshold (second part of (10)). Two different concepts can be used to measure the quality of the data sent to the sink: Pearson's coefficient of variation and Information Entropy.

- Pearson's coefficient of variation $(C V)$ is a rough measure of relative dispersion. The value of this coefficient for a group $g_{k}$ is $C V\left(g_{k}\right)=\frac{\sigma_{k}}{\bar{x}_{k}}$.

- Information entropy is a measure of the uncertainty or noise of a random variable. Applying this concept to this problem implies considering a group's entropy an indicator of the dissimilarity among the different group components. The information entropy associated to a generic group $g_{k}$ is given by $H\left(g_{k}\right)=\ln \left(\sigma_{k} \sqrt{2 \pi e}\right)$.

According to these two concepts, the quality of the information of the system for a certain configuration can be expressed in two different ways.

$$
\left.Q o I(t)\rfloor_{H}=\left(\sum_{\substack{c^{t} \in C_{\alpha} \\ c^{t}=\left\{g_{1}^{t}, \ldots, g_{n}^{t}\right\}}} \frac{H\left(g_{k}^{t}\right)}{n^{t}}\right)^{-1} ; Q o I(t)\right\rfloor_{C V}=\left(\sum_{\substack{c^{t} \in C_{\alpha} \\ c^{t}=\left\{g_{1}^{t}, \ldots, g_{n}^{t}\right\}}} \frac{C V\left(g_{k}^{t}\right)}{n^{t}}\right)^{-1}
$$




\subsection{Agents' Coalition Formation}

A group in a coalition structure acts as an entity. The leader of the group senses and sends a groupal value to the sink. If this value is a good representative of the variable value in the region covered by the group then, the group, as a whole, saves energy and computational resources.

Group formation is based on a peer-to-peer negotiation protocol by means of which agents exchange information about their measurements (adherence) and their adequacy to represent their neighbours (leadership).

The adherence degree of an agent $i$ to an agent $j$ is a measure that indicates how much agent $i$ intends to form part of a group led by agent $j$. The higher the degree, the higher the intention. The adherence degree is defined as the product of two factors. To evaluate those factors, we assume that the variable under observation follows a Normal distribution. Every agent $i$ knows an initial approximation of that distribution, $\mathcal{N}_{i}$. This distribution is updated by agent $i$ as it collects new samples, $\mathcal{N}_{i}\left(f\left(\bar{x}_{i}, x_{i}\right), g\left(\sigma_{i}, x_{i}\right)\right.$ ) (for appropriate $f$ and $g$ functions).

The first factor in the adherence expression (3) captures the similarity between the measurements of agents $i$ and $j$. It is defined as the quotient between the probability that the measure of an agent comes from the distribution of the neighbour (which could then be considered as the same value, perhaps with some noise) normalised by the maximum probability reachable in that distribution. To avoid unproductive computation, the similarity factor is only defined for neighbour agents that verify that $\left\|x_{j}-x_{i}\right\| \leq d_{\max } \sigma_{j}$, where $d_{\max }$ is a parameter and $x_{i}, \sigma_{j}$ are the corresponding sample and deviation of agents $i$ and $j$. On the other hand, the second factor captures the goodness of the neighbour's distribution and avoids obtaining high adherence values to neighbours with wide distributions. To get this, this factor restricts the evaluation to those neighbours whose $\sigma$ belongs to the interval $\left(\sigma_{\min }, \sigma_{\max }\right)$ through the evaluation of the distribution's entropy normalized on that range.

As a result, the evaluation of the degree to which an agent $a_{i}$ may be interested in being led by one of its neighbours $a_{j}$ is calculated as follows:

$$
\operatorname{adh}\left(a_{i}, a_{j}\right)=\frac{p\left(x_{i}, \mathcal{N}_{j}\left(\bar{x}_{j}, \sigma_{j}\right)\right)}{p\left(\bar{x}_{j}, \mathcal{N}_{j}\left(\bar{x}_{j}, \sigma_{j}\right)\right)} \cdot\left(1-\frac{e^{H_{j}}-e^{H_{\text {min }}}}{e^{H_{m a x}}-e^{H_{\text {min }}}}\right)
$$

Note that the set of $p$ parameters, as presented previously, can be identified now as $p=\left\langle d_{\max }, \sigma_{\min }, \sigma_{\max }\right\rangle$ defined over the space $p \in \mathbb{R}^{3}$. The set of values to which these parameters are set influence the actions that a node can take.

When an agent receives an adherence value from a neighbour, it has to decide whether it is interested in becoming the leader of this agent or not. Let us call $P\left(a_{i}\right)$ (potential group) the group formed by $a_{i}$ and the agents willing to become part of a group led by $a_{i}$. The attitude of $a_{i}$ as a leader of this group depends on different factors that can be identified in (4). The first factor is called prestige and it is an average of the adherence level of the group's members. The capacity factor indicates the available energy of the node to act as a leader. This value is derived from the current energy level of the node minus the security energy 
level $\left(E_{s l}\right)$ divided by the maximum energy level available $E_{\max } . E_{s l}$ defines the minimum energy that the node has to keep to ensure sending one last message before completely depleting its battery.

Finally, the last factor in (4), representativeness, indicates how well the potential leader's measurement fits as a representative of the potential group agents' measurements. So, $a_{i}$ characterizes the set of data received together with its own data, that is, the set $\{x\}_{P\left(a_{i}\right)}$, with their mean and standard deviation, noted as $\left(\bar{x}_{P\left(a_{i}\right)}, \sigma_{P\left(a_{i}\right)}\right)$. To encourage the formation of groups with very similar measurements, an exponential function establishes the divergence growing ratio: the larger the difference between the leader sample and the mean, the larger the penalty. Those potential groups whose measurement distribution is very disperse are also penalized through the inclusion of the Pearson's coefficient in the equation.

A good group leader is an agent who has enough energy and whose measurements are similar enough to the measurements of the other group members. In summary, the leadership capacity of an agent $a_{i}$ for its potential group $P\left(a_{i}\right)$ is calculated as follows:

$$
\operatorname{lead}\left(a_{i}, P\left(a_{i}\right)\right)=\frac{\sum_{a_{j} \in P\left(a_{i}\right)} a d h\left(a_{j}, a_{i}\right)}{N} \cdot \frac{E\left(a_{i}\right)-E_{s l}}{E_{\max }} \cdot \frac{1}{e^{\left|x_{i}-\bar{x}_{P\left(a_{i}\right)}\right| C V_{P\left(a_{i}\right)}}}
$$

\section{Operational Protocol}

Agents' preferences for coalition formation change due to the dynamics of the environment and the dynamics of the sensors. Based on the values of adherence and leadership, agents negotiate to form groups, trying to achieve their most preferred configuration at each time. The default situation is that of every agent alone constituting a group by itself (led by itself). In this section, we describe the algorithms that underpin the behaviour of the agents.

\subsection{Coalition Formation Protocol}

The coalition formation algorithm that all nodes execute can be divided in four processes that run simultaneously:

- Sample information exchange. This process corresponds to the variable sampling and measurements broadcast.

- Adherence graph construction. Once the agent has calculated the adherence degrees to its neighbours, it communicates the maximum adherence value to the corresponding most preferred neighbour.

- Leadership information exchange. Based on the current adherence relationships, the agent calculates and communicates its attitude as a leader towards the agents willing to adhere to it. 
- Group definition. Depending on the information available for an agent at a certain moment, it decides whether to stay in its current group (as a leader or dependant of a leader node), to leave this group to join a different one or to constitute its own group.

The messages exchanged between sensors in this negotiation follow a classical agent communication format: performative(sender, addresse(s), msgContent, [time]). The time field is an optional item that is used depending on the kind of performative. The set of performatives that the agents use are:

- inform: to indicate the transmission of data (measurements, maximum adherence and leadership values).

- firmAdherence: to express the desire of the sending node to adhere to the addressee node.

- ackAdherence: to express the acknowledgment to a previously received firmAdherence message.

- break: for a leader node to break a leadership relationship.

- withdraw: for a dependant node to break a leadership relationship.

Note that within the first three processes listed before, only the inform performative is used, while the rest of the performatives are used within the group definition process.

\subsection{Generic Agent Behaviour}

From an external point of view, it can be said that agents behave in a proactive and reactive way. Proactive because the core behaviour of an agent is the continuous process of looking for the best group of neighbours that matches with its measurement and its state. To achieve this objective, an agent exchanges messages asynchronously with its neighbours. Reactive because their acts and decisions are triggered by the observation of the environment and the information they receive.

The CF protocol is embedded in the agent behaviour via the execution of the Information Processing thread and the actions corresponding to the agent role at any moment (leader or dependant). The role changes along time depending on the information available at a certain moment (collected and processed through the Information Processing thread). When an agent is a leader, it starts sampling the environment and sending the measure to both its neighbours and the sink. Awaken agents communicate with each other to find an adequate configuration in which some of them may end up asleep for a preestablished time. Leader agents continue sensing and sending data to the sink according to the frequency demanded by the application, but this time, they work on behalf of their depending neighbours. When an agent is not a leader, it can, according to a certain probability, periodically take part in the CF configuration process together with all leader agents. This way it can change its state to become a leader of itself and others, depending on its current conditions. 


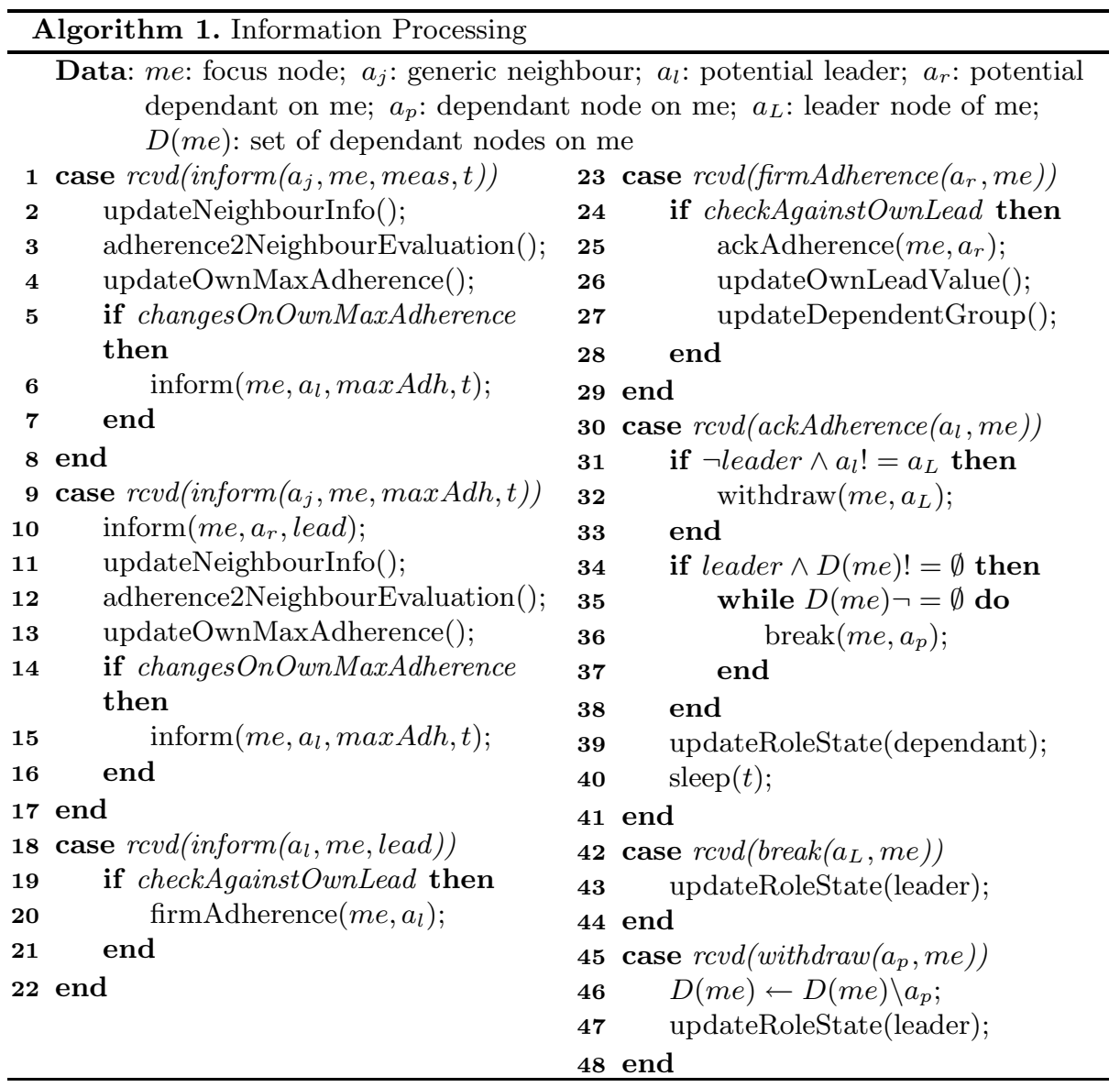

The core of the CF process is contained in Algorithm 1. This algorithm has been designed following a simple reactive structure in which all the actions are triggered by an event that changes the available information of an agent. This perspective allows the agent to decouple the different negotiation dialogues it may be involved in and also the different stages of each process. From a global point of view, information flows among the agents and it is this flow that makes agents react.

\subsection{Example of Coalition Formation}

Figures 113 illustrate how the algorithm works and some of the different coalition structures it may originate for a simple scenario of three agents. We start the illustration in a point where each agent has already been informed of the adherence preferences of its neighbours. Figure 1 shows the adherence graph where each agent is linked to its most preferred neighbour at a certain instant. For instance, agent $a_{1}$ has informed $a_{2}$ about its maximum adherence. The 


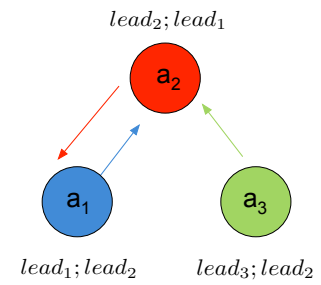

Fig. 1. Adherence relationships and lead information at each node

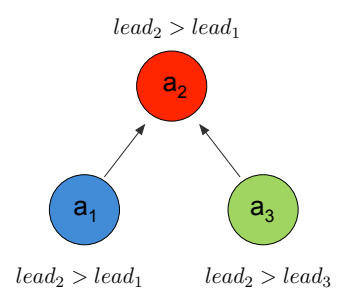

Fig. 2. Lead information evaluation and firm adherence messages emission

establishment of an adherence relationship implies enriching the information available for an agent with the leadership attitude of its preferred neighbour (Algorithm 11 line 10). So agent $a_{2}$ sends its current leadership attitude to $a_{1}$ and $a_{3}$. Agent $a_{2}$ receives the lead valueof $a_{1}$ and $a_{3}$ does not communicate its lead attitude to any other agent because no one wants to adhere to it. Once an agent gets the information about its neighbours' lead attitude, it decides whether it stands by this relationship or not (lines $18-22$ ). This decision is reached by comparing its own lead value to the neighbour's one (Fig. 2). Depending on the agent's current role (leader or dependant) this comparison could be done with different thresholds to encourage different desired behaviour of the overall WSN, i.e., agents could be more or less reluctant to changes or demanding on their neighbours' leadership strength 11 As a result of these comparisons, agents still willing to adhere to a neighbour send formal adherence messages that may or may not be answered by their potential leaders. When a potential leader receives a firm adherence message, it checks if it would be as good leader of the expanded group as it is of its current group. If so, it adds this new agent to its group, updates its lead attitude and sends an acknowledgement message to the new node to put it to sleep. On the other hand, if the agent's leadership would decrease by accepting the new neighbour, the agent will not answer to the potential new dependant, that may continue looking for a group to join (lines $23-29)$.

Different group configurations can be reached depending on the sequence of messages exchanged and the order in which they are received. Figure 3 shows three possible coalition structure configurations (with different number of groups each) reachable for this example. Each group of the coalition structure is described as a tuple formed by the leader and its set of dependent agents.

In the same way that coalition groups are formed, they can be broken by a leader or dependant agents (lines $42-48$ in Algorithm 1) when new information about neighbours is recieved or when its internal state (e.g. remaining energy) makes the agent's preferences change (e.g. joining a different group).

${ }^{1}$ The addition of these thresholds would imply changing the set of parameters $p$ to include them and also changing its definition domain. 


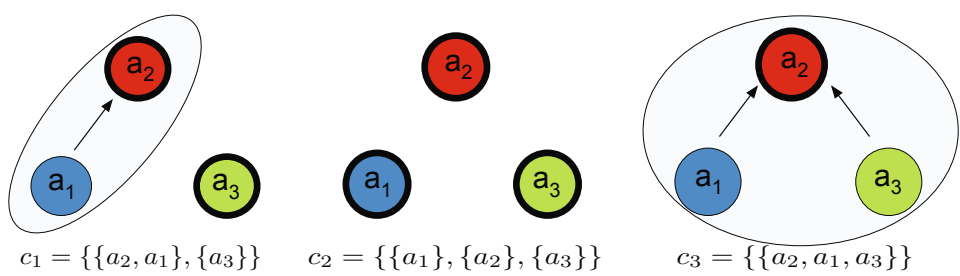

Fig. 3. Three possible resulting coalitions from the situation depicted in Fig. 2

It cannot be said that agents take their decisions and actions based on the actual situation of the environment (as such information may not be available), but on the current information about the environment. An agent is continuously involved in different negotiation processes with all its awaken neighbours and the decisions it takes are based on its most recent information. The intuition of the system function is that the more information an agent gets about what is happening, the more beneficial the coalition will be.

\section{Conclusions and Future Work}

In this work we have formalised and modelled the problem of coalition formation among distributed agents in a sensor network monitoring a wide area. For the considered scenario, agents join in coalitions in order to trade-off the quality of the sensed values and the life span of the network as a whole. The proposed distributed algorithm specifies the behaviour of the individual agents to accomplish their tasks and reach an appropriate group organisation. The algorithm's dynamics entail a changing distributed sampling of the environment, where the number of samples sent to the sink node depends on the current coalition structure of the network. The proposed algorithm is highly tuneable and eases the addition of an external control module on it. Currently, the experimentation to test the protocol is ongoing. Future work includes the extension of the approach over different sampling variable distribution models and different neighbourhood topologies. The influence of the social network derived from the preference graph structure on the topology and coalition distribution has also to be further explored. Finally, the comparison of the results of the algorithms for the two previously presented problem formulations (the first one based on Pearson's coefficient and the second one based on Information Entropy) will allow to identify which of the models better fits the experimental solution of the problem studied.

Acknowledgments. This work has been supported by the Agreement Technologies project (funded by CONSOLIDER CSD 2007-0022, INGENIO 2010). 


\section{References}

1. Akyildiz, I.F., Su, W., Sankarasubramaniam, Y., Cayirci, E.: A survey on sensor networks. IEEE Communications Magazine 40(8), 102-114 (2002)

2. Bai, Q., Zhang, M.: Rational, Robust, and Secure Negotiations in Multi-Agent Systems. In: A Fuzzy Logic-Based Approach for Flexible Self-Interested Agent Team Forming. SCI, vol. 89, pp. 101-113. Springer, Heidelberg (2008)

3. Barton, L., Allan, V.H.: Methods for Coalition Formation in Adaptation-Based Social Networks. In: Klusch, M., Hindriks, K.V., Papazoglou, M.P., Sterling, L. (eds.) CIA 2007. LNCS (LNAI), vol. 4676, pp. 285-297. Springer, Heidelberg (2007)

4. Gasser, L.: Social knowledge and social action: heterogeneity in practice. In: Proceedings of the 13th International Joint Conference on Artifical Intelligence, vol. 1, pp. 751-757. Morgan Kaufmann Publishers Inc., San Francisco (1993)

5. Gaston, M.E., des Jardins, M.: Agent-organized networks for dynamic team formation. In: Proceedings of the 4th International Conference on Autonomous Agents and Multiagent Systems, AAMAS 2005, pp. 230-237. ACM, New York (2005)

6. Glinton, R., Scerri, P., Sycara, K.: Agent-based sensor coalition formation. In: 2008 11th International Conference on Information Fusion, pp. 1-7 (July 2008)

7. Griffiths, N., Luck, M.: Coalition formation through motivation and trust. In: Proceedings of the 2nd International Conference on Autonomous Agents and Multiagent Systems, AAMAS 2003, pp. 17-24. ACM, New York (2003)

8. Kraus, S., Shehory, O., Taase, G.: Coalition formation with uncertain heterogeneous information. In: Proceedings of the 2nd International Conference on $\mathrm{Au}$ tonomous Agents and Multiagent Systems, AAMAS 2003, pp. 1-8. ACM, New York (2003)

9. Mac Ruairí, R., Keane, M.T.: The dynamic regions theory: Role based partitioning for sensor network optimization. In: Proceedings of the 6th International Conference on Autonomous Agents and Multiagent Systems (2007)

10. Mas-Colell, A., Whinston, M.D., Green, J.R.: Microeconomic Theory. Oxford University Press (June 1995)

11. Padhy, P., Dash, R.K., Martinez, K., Jennings, N.R.: A utility-based sensing and communication model for a glacial sensor network. In: Proceedings of the 5th International Conference on Autonomous Agents and Multiagent Systems, AAMAS 2006, pp. 1353-1360. ACM, New York (2006)

12. Sims, M., Goldman, C.V., Lesser, V.: Self-organization through bottom-up coalition formation. In: Proceedings of the 2nd International Conference on $\mathrm{Au}-$ tonomous Agents and Multiagent Systems, AAMAS 2003, pp. 867-874. ACM, New York (2003)

13. Smith, R.G.: The contract net protocol: High-level communication and control in a distributed problem solver. IEEE Transactions on Computers C-29(12), 1104-1113 (1980)

14. Vig, L., Adams, J.A.: Coalition formation: From software agents to robots. J. Intell. Robotics Syst. 50, 85-118 (2007) 